\title{
The Incremental Harmonic Balance Method for Nonlinear Vibration of Axially Moving Beams
}

\author{
K.Y.Sze* \\ Department of Mechanical Engineering, The University of Hong Kong, \\ Pokfulam Road, Hong Kong SAR, P.R.CHINA. \\ and \\ S.H.Chen, J.L.Huang \\ Department of Applied Mechanics and Engineering, Zhongshan University \\ Guangzhou, 510275, P.R.CHINA.
}

\begin{abstract}
In this paper, the Incremental Harmonic Balance (IHB) method is formulated for the nonlinear vibration analysis of axially moving beams. The Galerkin method is used to discretize the governing equations. A high dimensional model that can take nonlinear model coupling into account is derived. The forced response of an axially moving strip with internal resonance between the first two transverse modes is studied. Particular attention is paid to the fundamental, superharmonic and subharmonic resonance as the excitation frequency is close to the first, second or one-third of the first natural frequency of the system. Numerical results reveal the rich and interesting nonlinear phenomena that have not been presented in the existent literature on the nonlinear vibration of axially moving media.
\end{abstract}

Keywords: axially moving, incremental harmonic balance method, nonlinear vibration

* Corresponding author. Email address : kysze@,hku.hk 


\section{INTRODUCTION}

Axially moving systems can be found in a wide range of engineering problems which arise in industrial, civil, mechanical, electronic and automotive applications. Magnetic tapes, power transmission belts and band saw blades are examples where an axial transport of mass is associated with a transverse vibration.

Analytical models for axially moving systems have been extensively studied in the last few decades. The vast literature on axially moving material vibration has been reviewed by Wickert and Mote [1] up to 1988. More recently, the problem of axially moving media has been tackled in the analysis of particular aspects such as different solution techniques, discretization approaches, modeling aspects and nonlinear phenomena, see the review in reference [2]. Most of these studies addressed the problem of constant axial transport velocity and constant axial tension. Wickert and Mote [3] studied the transverse vibration of axially moving strings and beams using an eigenfunction method. They also used the Green function method to study the dynamic response of an axially moving string loaded with a traveling periodic suspended mass [4]. Wickert [5] presented a complete study of the nonlinear vibrations and bifurcations of moving beams using the KrylovBogoliubov-Mitropolsky asymptotic method. Chakraborty et al [6,7] investigated both free and forced responses of a traveling beam using non-linear complex normal modes. Pellicano et al [2] studied the dynamic behavior of an axially moving beam using a high dimensional discrete model obtained by the Galerkin procedure. Al-Jawi et al. [8-10] investigated the effects of tension disorder, inter-span coupling and translational speed on the confinement of the natural modes of free vibration through the exact, the perturbation and the Galerkin approaches. By analytical and numerical means, Chen [11] studied the natural frequencies and stability of an axially traveling string in contact with a stationary load system which contains parameters such as dry friction, inertia, damping and stiffness. Riedel and Tan [12] studied the coupled and forced responses of an axially moving strip with internal resonance. The method of multiple scales is used to perform the perturbation analysis and to determine the frequency response numerically for both low and high speeds.

There are papers devoted to the analysis of the dynamic behavior of traveling systems with time dependent axial velocity or with time dependent axial tension force. Pakdemirli et al. [13] conducted a stability analysis using Floquet theory for sinusoidal transporting velocity function. They also investigated the principal parametric resonance and the combination of resonances for an axially accelerating string using the method of multiple scales [14]. Mockensturm et al. [15] applied the Galerkin procedure and the perturbation method of Krylov-Bogoliubov-Mitropolsky to examine the stability and limit cycles of parametrically excited and axially moving strings in the presence of 
tension fluctuations. Zhang and $\mathrm{Zu}[16,17]$ employed the method of multiple scales to study the nonlinear vibration of parametrically excited moving belts. Suweken and Van Horssen [18] used a two time-scales perturbation method to approximate the solutions of a conveyor belt with a low and time-varying velocity. Öz and Pakdemirli [19,20] also applied the method of multiple scales to study the vibration of an axially moving beam with time dependent velocity. Fung and Chang [21] employed the finite difference method with variable grid for numerical calculation of string/slider non-linear coupling system with time-dependent boundary condition. Ravindra and Zhu [22] studied the low-dimensional chaotic response of axially accelerating continuum in the supercritical regime. Moon and Wickert [23] performed an analytical and experimental study on the response of a belt excited by pulley eccentricities. Pellicano et al [24] studied the primary and parametric nonlinear resonance of a power transmission belt by experimental and theoretical analysis.

In this paper, the Incremental Harmonic Balance (IHB) method is applied to analyze the nonlinear vibration of axially moving systems. The IHB method was originally presented by Lau and Cheung [25], Cheung and Lau [26] and Lau, Cheung and $\mathrm{Wu}$ [27]. It has been developed and successfully applied to the analysis of periodic nonlinear structural vibrations and the related problems. However, none of these applications is related to axially moving systems. This paper starts with an introduction on the essence of the IHB method. Using the method, some particular cases of the axially moving beam problem are effectively treated. As a matter of fact, the generalization of the IHB method to other moving media and other nonlinear vibration problems is simple and straightforward.

\section{EQUATIONS OF MOTION}

The governing equations of two dimensional, planar motion of an axially moving beam can be derived using Hamilton's Principle. Following a similar derivation to that of Wickert [5], one obtains the following coupled nonlinear dimensionless equations of motion:

$$
\begin{gathered}
\left(u,_{t t}+2 v u,_{x t}+v^{2} u,_{x x}\right)-v_{1}^{2}\left(u,_{x}+\frac{1}{2} w,_{x}^{2}\right),_{x}=0 \\
\left(w,_{t t}+2 v w,_{x t}+v^{2} w,_{x x}\right)-\left\{\left[1+v_{1}^{2}\left(u,_{x}+\frac{1}{2} w,_{x}^{2}\right)\right] w,_{x}\right\},_{x}+v_{f}^{2} w,_{x x x x}=0
\end{gathered}
$$

where

$$
\begin{gathered}
u=U / L, \quad w=W / L, \quad x=X / L, \quad t=T \sqrt{P / \mathrm{r} A L^{2}} \\
v=V / \sqrt{P / \mathrm{r} A}, v_{1}=\sqrt{E A / P}, v_{f}=\sqrt{E I / P L^{2}}
\end{gathered}
$$

Here, $X$ and $Z$ are respectively the longitudinal and transverse coordinates of the beam, $U$ and $W$ are respectively the longitudinal and transverse displacements, $V$ is the axial speed, $T$ denotes time and 
$P$ is the axial tension, see Figure 1. Properties of the beam include the beam length $L$, the cross section area $A$, the second moment of area $I$, the mass density $\mathrm{r}$ and the elastic modulus $E$. Lastly, $\left(u,_{t t}, w_{t_{t}}\right),\left(2 v u,_{x t}, 2 v w,_{x t}\right)$ and $\left(v^{2} u,_{x x}, v^{2} w,_{x x}\right)$ are respectively the local, Coriolis and centripetal acceleration vectors. In particular, $V, P, L, A, I, \mathrm{r}$ and $I$ are constants whereas $P$ must be non-zero. The boundary conditions for a hinged-hinged beam are:

$$
\begin{gathered}
u(0, t)=u(1, t)=0, \\
w(0, t)=w(1, t)=0, w,_{x x}(0, t)=w,_{x x}(1, t)=0 .
\end{gathered}
$$

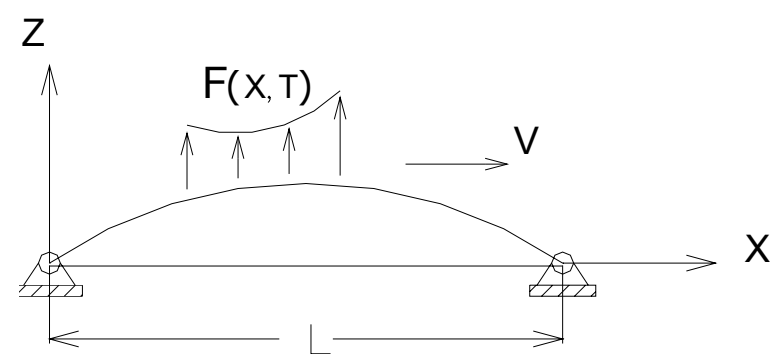

Figure 1. Schematic diagram for an axially moving beam and its coordinate system, where $\mathrm{F}$ denotes the force acting per unit length of the beam.

\section{SEPARATION OF VARIABLES}

In the nonlinear vibration analysis of continuous systems, the variables $u(x, t)$ and $w(x, t)$ in the partial differential equations (1) and (2) with boundary conditions (5) and (6) are usually separated. The use of eigenfunctions as a complete basis is often the choice because of their promising accuracy and convergence. Unfortunately, the eigenfunctions of traveling beams are complex and dependent on the speed. In the present study, we assume the following separable solutions in terms of admissible functions:

$$
\begin{aligned}
& u(x, t)=\sum_{j=1}^{N} q_{j}^{u}(t) \sin j \mathrm{p} x, \\
& w(x, t)=\sum_{j=1}^{N} q_{j}^{w}(t) \sin j \mathrm{p} x .
\end{aligned}
$$

After substituting equations (7) and (8) into equations (1) and (2), the Galerkin procedure leads to the following set of $N+M$ second order ordinary differential equations:

$$
\sum_{j=1}^{N} M_{i j}^{u} \ddot{q}_{j}^{u}+\sum_{j=1}^{N} C_{i j}^{u} \dot{q}_{j}^{u}+\sum_{j=1}^{N} K_{i j}^{u} q_{j}^{u}+\sum_{j=1}^{N} \sum_{k=1}^{M} K_{i j k}^{w} q_{k}^{w} q_{j}^{w}=0 \quad \text { for } i=1,2, \cdots, N
$$




$$
\begin{array}{r}
\sum_{j=1}^{M} M_{i j}^{w} \ddot{q}_{j}^{w}+\sum_{j=1}^{M} C_{i j}^{w} \dot{q}_{j}^{w}+\sum_{j=1}^{M} K_{i j}^{w} q_{j}^{w}+\sum_{j=1}^{M} \sum_{k=1}^{N} K_{i j k}^{u} q_{k}^{u} q_{j}^{w}+\sum_{j=1}^{M} \sum_{k=1}^{M} \sum_{l=1}^{M} K_{i j k l}^{w} q_{l}^{w} q_{k}^{w} q_{j}^{w}=0 \\
\text { for } i=1,2, \cdots, M
\end{array}
$$

where

the dot above a variable denotes its derivative with respect to the non-dimensional time $t$,

$$
\begin{aligned}
& M_{i j}^{u}=M_{i j}^{w}=\int_{0}^{1} \sin i \mathrm{p} x \sin j \mathrm{p} x \mathrm{~d} x=\frac{1}{2} \delta_{i j}, \\
& K_{i j}^{u}=-\left(v^{2}-v_{1}^{2}\right) j^{2} \mathrm{p}^{2} \int_{0}^{1} \sin i \mathrm{p} x \sin j \mathrm{p} x \mathrm{~d} x=-\frac{1}{2}\left(v^{2}-v_{1}^{2}\right) j^{2} \mathrm{p}^{2} \mathrm{~d}_{i j}, \\
& C_{i j}^{u}=C_{i j}^{w}=2 v j \mathrm{p} \int_{0}^{1} \sin i \mathrm{p} x \cos j \mathrm{p} x \mathrm{~d} x= \begin{cases}4 i j v /\left(i^{2}-j^{2}\right) & i \neq j \text { and }(i+j) \text { is even } \\
0 & \text { otherwise }\end{cases} \\
& K_{i j k}^{w}=v_{1}^{2} j k^{2} \mathrm{p}^{3} \int_{0}^{1} \sin i \mathrm{p} x \cos j \mathrm{p} x \sin k \mathrm{p} x \mathrm{~d} x=v_{1}^{2} j k^{2} \mathrm{p}^{3} I_{s c s}(i, j, k), \\
& K_{i j}^{w}=\left[v_{f}^{2} j^{4} \mathrm{p}^{4}-\left(v^{2}-1\right) j^{2} \mathrm{p}^{2}\right] \int_{0}^{1} \sin i \mathrm{p} x \sin j \mathrm{p} x \mathrm{~d} x=\frac{1}{2}\left[v_{f}^{2} j^{4} \mathrm{p}^{4}-\left(v^{2}-1\right) j^{2} \mathrm{p}^{2}\right] \mathrm{d}_{i j}, \\
& K_{i j k}^{u}=v_{1}^{2} j k^{2} \mathrm{p}^{3} \int_{0}^{1} \sin i \mathrm{p} x \cos j \mathrm{p} x \sin k \mathrm{p} x \mathrm{~d} x+v_{1}^{2} k j^{2} \mathrm{p}^{3} \int_{0}^{1} \sin i \mathrm{p} x \cos k \mathrm{p} x \sin j \mathrm{p} x \mathrm{~d} x, \\
& =v_{1}^{2} j k^{2} \mathrm{p}^{3} I_{s c s}(i, j, k)+v_{1}^{2} k j^{2} \mathrm{p}^{3} I_{s c s}(i, k, j), \\
& K_{i j k l}^{w}=\frac{3}{2} v_{1}^{2} j^{2} k l \mathrm{p}^{4} \int_{0}^{1} \sin i \mathrm{p} x \sin j \mathrm{p} x \cos k \mathrm{p} x \cos l \mathrm{p} x \mathrm{~d} x=\frac{3}{2} v_{1}^{2} j^{2} k l \mathrm{p}^{4} I_{s s c c}(i, j, k, l), \\
& I_{s c c}(i, j)=\int_{0}^{1} \cos i \pi x \cos j \pi x \mathrm{~d} x= \begin{cases}0 & i \neq j \\
1 / 2 & i=j \neq 0 \\
1 & i=j=0\end{cases} \\
& I_{s s c c}(i, j, k, l)=\frac{1}{4}\left[I_{c c}(i-j, k+l)+I_{c c}(i-j, k-l)-I_{c c}(i+j, k+l)-I_{c c}(i+j, k-l)\right], \\
& \left.I_{c c}(i-k, j)-I_{c c}(I+k, j)\right],
\end{aligned}
$$

Equations (9) and (10) can be written in matrix-vector form as:

$$
\begin{gathered}
\mathbf{M}^{\mathrm{u}} \ddot{\mathbf{q}}^{\mathrm{u}}+\mathbf{C}^{\mathrm{u}} \dot{\mathbf{q}}^{\mathrm{u}}+\mathbf{K}^{\mathrm{u}} \mathbf{q}^{\mathrm{u}}+\mathbf{K}_{2}^{\mathrm{w}}\left(\mathbf{q}^{\mathrm{w}}\right) \mathbf{q}^{\mathrm{w}}=\mathbf{0}, \\
\mathbf{M}^{\mathrm{w}} \ddot{\mathbf{q}}^{\mathrm{w}}+\mathbf{C}^{\mathrm{w}} \dot{\mathbf{q}}^{\mathrm{w}}+\mathbf{K}^{\mathrm{w}} \mathbf{q}^{\mathrm{w}}+\mathbf{K}_{2}^{\mathrm{u}}\left(\mathbf{q}^{\mathrm{u}}\right) \mathbf{q}^{\mathrm{w}}+\mathbf{K}_{3}^{\mathrm{w}}\left(\mathbf{q}^{\mathrm{w}}\right) \mathbf{q}^{\mathrm{w}}=\mathbf{0}
\end{gathered}
$$

where $\mathbf{q}^{\mathrm{u}}=\left[q_{1}^{u}, q_{2}^{u}, \cdots, q_{N}^{u}\right]^{\mathrm{T}}$ and $\mathbf{q}^{\mathrm{w}}=\left[q_{1}^{w}, q_{2}^{w}, \cdots, q_{M}^{w}\right]^{\mathrm{T}}$. The entries of matrices $\mathbf{M}^{\mathrm{u}}, \mathbf{M}^{\mathrm{w}}, \mathbf{C}^{\mathrm{u}}, \mathbf{C}^{\mathrm{w}}$, $\mathbf{K}^{\mathrm{u}}$ and $\mathbf{K}^{\mathrm{w}}$ are respectively $M_{i j}^{u}, M_{i j}^{w}, C_{i j}^{u}, C_{i j}^{w}, K_{i j}^{u}$ and $K_{i j}^{w}$. Furthermore, the entries of matrices $\mathbf{K}_{2}^{\mathrm{w}}\left(\mathbf{q}^{\mathrm{w}}\right), \mathbf{K}_{2}^{\mathrm{u}}\left(\mathbf{q}^{\mathrm{u}}\right)$ and $\mathbf{K}_{3}^{\mathrm{w}}\left(\mathbf{q}^{\mathrm{w}}\right)$ are respectively 


$$
K_{2_{i j}}^{w}=\sum_{k=1}^{M} k_{i j k}^{w} q_{k}^{w}, \quad K_{2_{i j}}^{u}=\sum_{k=1}^{N} K_{i j k}^{u} q_{k}^{u} \quad \text { and } \quad K_{3_{i j}}^{w}=\sum_{k=1}^{M} \sum_{l=1}^{M} K_{i j k l}^{w} q_{k}^{w} q_{l}^{w} .
$$

Equations (11) and (12) can be grouped as:

$$
\mathbf{M} \ddot{\mathbf{q}}+\mathbf{C} \dot{\mathbf{q}}+\mathbf{K q}+\mathbf{K}_{2}(\mathbf{q}) \mathbf{q}+\mathbf{K}_{3}(\mathbf{q}) \mathbf{q}=\mathbf{0}
$$

where

$$
\begin{aligned}
& \mathbf{q}=\left[\mathbf{q}^{\mathrm{u}}, \mathbf{q}^{\mathrm{w}}\right]^{\mathrm{T}}, \mathbf{M}=\left[\begin{array}{cc}
\mathbf{M}^{\mathrm{u}} & \mathbf{0} \\
\mathbf{0} & \mathbf{M}^{\mathrm{w}}
\end{array}\right], \mathbf{C}=\left[\begin{array}{cc}
\mathbf{C}^{\mathrm{u}} & \mathbf{0} \\
\mathbf{0} & \mathbf{C}^{\mathrm{w}}
\end{array}\right], \\
& \mathbf{K}=\left[\begin{array}{cc}
\mathbf{K}^{\mathrm{u}} & \mathbf{0} \\
\mathbf{0} & \mathbf{K}^{\mathrm{w}}
\end{array}\right], \mathbf{K}_{2}=\left[\begin{array}{cc}
\mathbf{0} & \mathbf{K}_{2}^{\mathbf{w}} \\
\mathbf{0} & \mathbf{K}_{2}^{\mathrm{u}}
\end{array}\right], \mathbf{K}_{3}=\left[\begin{array}{cc}
\mathbf{0} & \mathbf{0} \\
\mathbf{0} & \mathbf{K}_{3}^{\mathrm{w}}
\end{array}\right] .
\end{aligned}
$$

For the forced response of the system, an excitation term can be added to the right hand side of equation (13), i.e.

$$
\mathbf{M} \ddot{\mathbf{q}}+\mathbf{C} \dot{\mathbf{q}}+\mathbf{K q}+\mathbf{K}_{2}(\mathbf{q}) \mathbf{q}+\mathbf{K}_{3}(\mathbf{q}) \mathbf{q}=\mathbf{F} \cos n w t
$$

in which $\mathrm{w}$ is the non-dimensional excitation frequency whose physical counterpart is $\mathrm{w} \sqrt{P / \mathrm{r} A L^{2}}$.

\section{IHB FORMULATION}

In this section, the Incremental Harmonic Balance (IHB) method is formulated to solve equation (14). With the new dimensionless time variable $t$ defined as:

$$
\mathrm{t}=\mathrm{w} t
$$

equation (14) becomes

$$
\mathrm{w}^{2} \mathbf{M} \mathbf{q}^{\prime \prime}+\mathrm{w} \mathbf{C q}^{\prime}+\left[\mathbf{K}+\mathbf{K}_{2}(\mathbf{q})+\mathbf{K}_{3}(\mathbf{q})\right] \mathbf{q}=\mathbf{F} \cos n t
$$

in which prime denotes differentiation with respect to $t$.

The first step of the IHB method is the incremental procedure. Let $q_{j 0}$ and $\mathrm{w}_{0}$ denote a state of vibration; the neighboring state can be expressed by adding the corresponding increments as:

$$
\mathrm{W}=\mathrm{w}_{0}+\mathrm{Dw}, q_{j}=q_{j 0}+\mathrm{D} q_{j}
$$

where $j=1,2, \cdots, m$ and $m=N+M$.

Substituting equations (17) into equation (16) and neglecting the higher order incremental terms, one obtains the following linearized incremental equation in matrix-vector form:

$$
\begin{aligned}
& \mathrm{W}_{0}^{2} \mathbf{M D q} \mathbf{q}^{\prime \prime}+\mathrm{w}_{0} \mathbf{C D q ^ { \prime }}+\left(\mathbf{K}+\mathbf{K}_{2}^{*}+3 \mathbf{K}_{3}\right) \mathrm{Dq}=\mathbf{R}-\left(2 \mathrm{w}_{0} \mathbf{M} \mathbf{q}_{0}^{\prime \prime}+\mathbf{C} \mathbf{q}_{0}^{\prime}\right) \mathrm{Dw}, \\
& \mathbf{R}=\mathbf{F} \cos n t-\left\{\mathrm{w}_{0}^{2} \mathbf{M} \mathbf{q}_{0}^{\prime \prime}+\mathrm{w}_{0} \mathbf{C} \mathbf{q}_{0}^{\prime}+\left[\mathbf{K}+\mathbf{K}_{2}\left(\mathbf{q}_{0}\right)+\mathbf{K}_{3}\left(\mathbf{q}_{0}\right)\right] \mathbf{q}_{0}\right\}
\end{aligned}
$$

in which 


$$
\begin{gathered}
\mathbf{q}_{0}=\left[q_{10}, q_{20}, \cdots, q_{m 0}\right]^{\mathrm{T}}, \mathrm{D} \mathbf{q}=\left[\mathrm{D} q_{1}, \mathrm{D} q_{2}, \cdots, \mathrm{D} q_{m}\right]^{\mathrm{T}}, \\
\mathbf{K}_{2}^{*}=\left[\begin{array}{cc}
\mathbf{0} & 2 \mathbf{K}_{\mathbf{2}}^{\mathbf{w}} \\
\mathbf{K}_{2}^{+} & \mathbf{K}_{2}^{\mathbf{u}}
\end{array}\right], K_{2_{i j}^{+}}^{+}=\sum_{k=1}^{M} K_{i j k}^{u} q_{k}^{w}
\end{gathered}
$$

and $\mathbf{R}$ is a residual/corrective vector that goes to zero when the (numerical) solution is exact.

The second step of the IHB method is the harmonic balance procedure. Let

$$
\begin{gathered}
q_{j 0}=\sum_{k=1}^{n_{c}} a_{j k} \cos (k-1) \mathrm{t}+\sum_{k=1}^{n_{s}} b_{j k} \sin k \mathrm{t}=\mathbf{C}_{s} \mathbf{A}_{j}, \\
\mathrm{D} q_{j}=\sum_{k=1}^{n_{c}} \mathrm{D} a_{j k} \cos (k-1) \mathrm{t}+\sum_{k=1}^{n_{s}} \mathrm{D} b_{j k} \sin k \mathrm{t}=\mathbf{C}_{s} \mathrm{D} \mathbf{A}_{j}
\end{gathered}
$$

where

$$
\begin{gathered}
\mathbf{C}_{s}=\left[1, \cos \mathrm{t}, \cdots, \cos \left(n_{c}-1\right) \mathrm{t}, \sin \mathrm{t}, \cdots, \sin n_{s} \mathrm{t}\right], \\
\mathbf{A}_{j}=\left[a_{j 1}, a_{j 2}, \cdots, a_{j n_{c}}, b_{j 1}, b_{j 2}, \cdots, b_{j n_{s}}\right]^{\mathrm{T}}, \\
\mathrm{DA}_{j}=\left[\mathrm{D} a_{j 1}, \mathrm{D} a_{j 2}, \cdots, \mathrm{D} a_{j n_{c}}, \mathrm{D} b_{j 1}, \mathrm{D} b_{j 2}, \cdots, \mathrm{D} b_{j n_{s}}\right]^{\mathrm{T}} .
\end{gathered}
$$

Then, $\mathbf{q}_{0}$ and Dq can be expressed in terms of the Fourier coefficient vector $\mathbf{A}=\left[\mathbf{A}_{1}, \mathbf{A}_{2} \cdots, \mathbf{A}_{m}\right]^{\mathrm{T}}$ and its increment $\mathrm{DA}=\left[\mathrm{DA} \mathbf{A}_{1}, \mathrm{DA}_{2} \cdots, \mathrm{DA}_{m}\right]^{\mathrm{T}}$ as

$$
\mathbf{q}_{0}=\mathbf{S A}, \mathrm{Dq}=\mathbf{S D A}
$$

in which $\mathbf{S}=\operatorname{diag} .\left[\mathbf{C}_{\mathrm{s}}, \mathbf{C}_{\mathrm{s}}, \cdots, \mathbf{C}_{\mathrm{s}}\right]$. Substituting equations (22) into equation (18) and applying the Galerkin procedure for one cycle, one obtains the following set of linear equations in terms of DA and Dw:

$$
\overline{\mathbf{K}}_{m c} \mathrm{DA}=\overline{\mathbf{R}}-\overline{\mathbf{R}}_{m c} \mathrm{Dw}
$$

where

$$
\begin{gathered}
\overline{\mathbf{K}}_{m c}=\int_{0}^{2 \mathrm{p}} \mathbf{S}^{\mathrm{T}}\left[\mathrm{w}_{0}^{2} \mathbf{M} \mathbf{S}^{\prime \prime}+\mathrm{w}_{0} \mathbf{C S}^{\prime}+\left(\mathbf{K}+\mathbf{K}_{2}^{*}+3 \mathbf{K}_{3}\right) \mathbf{S}\right] d t \\
\overline{\mathbf{R}}=\int_{0}^{2 \mathrm{p}} \mathbf{S}^{\mathrm{T}}\left\{\mathbf{F} \cos n t-\left[\mathrm{w}_{0}^{2} \mathbf{M} \mathbf{S}^{\prime \prime}+\mathrm{w}_{0} \mathbf{C S}^{\prime}+\left(\mathbf{K}+\mathbf{K}_{2}+\mathbf{K}_{3}\right) \mathbf{S}\right]\right\} d t \mathbf{A}, \\
\overline{\mathbf{R}}_{m c}=\int_{0}^{2 \mathrm{p}} \mathbf{S}^{\mathrm{T}}\left(2 \mathrm{w}_{0} \mathbf{M S}^{\prime \prime}+\mathbf{C S}^{\prime}\right) d \mathrm{t} \mathbf{A} .
\end{gathered}
$$

The solution process begins with a guessed solution. The nonlinear frequency-amplitude response curve is then solved point-by-point by incrementing the frequency $\mathrm{w}$ or incrementing a component of the coefficient vector A. The Newton-Raphson iterative method can be employed. 


\section{NUMERICAL CALCULATIONS}

To illustrate the power of the IHB method, some numerical examples are presented in this section. If we take $N=M=1$ in equations (7) and (8), i.e., only one longitudinal mode and one transverse mode are taken, then equations (9) and (10) become

$$
\begin{gathered}
\ddot{q}_{1}^{u}-\left(v^{2}-v_{1}^{2}\right) \mathrm{p}^{2} q_{1}^{u}=0, \\
\ddot{q}_{1}^{w}+\left(v_{f}^{2} \mathrm{p}^{2}-v^{2}+1\right) \mathrm{p}^{2} q_{1}^{w}+\frac{3}{8} v_{1}^{2} \mathrm{p}^{4}\left(q_{1}^{w}\right)^{3}=0 .
\end{gathered}
$$

Obviously, the longitudinal vibration is linear and the transverse vibration is nonlinear. Moreover, they are not coupled. Equation (25) is the famous Duffing equation and its nonlinear dynamic characteristics have been thoroughly investigated by many researchers. Letting $N=M=2$, i.e. two longitudinal and two transverse modes are considered, equations (9) and (10) become:

$$
\begin{gathered}
\ddot{q}_{1}^{u}+\mathrm{m}_{1} \dot{q}_{2}^{w}-\left(v^{2}-v_{1}^{2}\right) \mathrm{p}^{2} q_{1}^{u}+v_{1}^{2} \mathrm{p}^{3} q_{1}^{w} q_{2}^{w}=0, \\
\ddot{q}_{2}^{u}+\mathrm{m}_{2} \dot{q}_{1}^{u}-4\left(v^{2}-v_{1}^{2}\right) \mathrm{p}^{2} q_{2}^{u}+\frac{1}{2} v_{1}^{2} \mathrm{p}^{3}\left(q_{1}^{w}\right)^{2}=0, \\
\ddot{q}_{1}^{w}+\mathrm{m}_{1} \dot{q}_{2}^{w}+k_{11} q_{1}^{w}+k_{12} q_{1}^{w}\left(q_{2}^{w}\right)^{2}+k_{13}\left(q_{1}^{w}\right)^{3}+v_{1}^{2} \mathrm{p}^{3}\left(q_{1}^{u} q_{2}^{w}+q_{2}^{u} q_{1}^{w}\right)=0, \\
\ddot{q}_{2}^{w}+\mathrm{m}_{2} \dot{q}_{1}^{w}+k_{21} q_{2}^{w}+k_{22} q_{2}^{w}\left(q_{1}^{w}\right)^{2}+k_{23}\left(q_{2}^{w}\right)^{3}+v_{1}^{2} \mathrm{p}^{3} q_{1}^{u} q_{1}^{w}=0
\end{gathered}
$$

where

$$
\begin{gathered}
\mathrm{m}_{1}=-16 v / 3, k_{11}=\left(v_{f}^{2} \mathrm{p}^{2}-v^{2}+1\right) \mathrm{p}^{2}, \quad k_{12}=3 v_{1}^{2} \mathrm{p}^{4}, \quad k_{13}=k_{12} / 8, \\
\mathrm{~m}_{2}=-\mathrm{m}_{1}, \quad k_{21}=4\left(4 v_{f}^{2} \mathrm{p}^{2}-v^{2}+1\right) \mathrm{p}^{2}, k_{22}=k_{12}, \quad k_{23}=2 k_{12} .
\end{gathered}
$$

Riedel and Tan [12] investigated the forced transverse response of an axially moving strip using the method of multiple scales. With reference to the typical parameters of a belt drive system given in [12], we shall assume

$$
v_{1}^{2}=1124, v_{f}^{2}=0.0015 \text { and } v=0.6
$$

throughout this section. The natural frequencies can be approximated with the linear undamped natural frequencies by dropping the nonlinear and damping terms in equations (26) to (29). In this light, the linear natural frequencies are estimated to be 2.54 and 5.25 for the transverse vibration, and 105.31 and 210.62 for the longitudinal vibration. The natural frequencies of the transverse vibration are far away from that of the longitudinal vibration so that the coupled effect between them should be weak. Hence, we will focus on the forced transverse response of the moving beam by neglecting the effect of the longitudinal vibration in the following study. By setting $q_{i}^{u}$ s to zero and by incorporating the force terms, equations (28) and (29) become

$$
\ddot{q}_{1}^{w}+\mathrm{m}_{1} \dot{q}_{2}^{w}+k_{11} q_{1}^{w}+k_{12} q_{1}^{w}\left(q_{2}^{w}\right)^{2}+k_{13}\left(q_{1}^{w}\right)^{3}=F_{1} \cos \mathrm{W} t
$$




$$
\ddot{q}_{2}^{w}+\mathrm{m}_{2} \dot{q}_{1}^{w}+k_{21} q_{2}^{w}+k_{22} q_{2}^{w}\left(q_{1}^{w}\right)^{2}+k_{23}\left(q_{2}^{w}\right)^{3}=F_{2} \cos \mathrm{W} t
$$

where $\Omega$ is the forcing frequency. By dropping the nonlinear terms, the linear natural frequencies can be solved from the following equation:

$$
\mathrm{w}^{4}-\left(k_{11}+k_{21}-\mathrm{m}_{1} \mathrm{~m}_{2}\right) \mathrm{w}^{2}+k_{11} k_{21}=0
$$

For the chosen $v_{1}$ and $v_{f}$, Figure 2 plots the ratio of the second natural frequency $\mathrm{w}_{20}$ to the first natural frequency $\mathrm{W}_{10}$ versus the axial speed $v$. For a nonlinear system with cubic nonlinearity, the internal resonance usually occurs when the natural frequency $\mathrm{w}_{20} \approx 3 \mathrm{w}_{10}$. It can be found from Figure 2 that $\mathrm{W}_{20} / \mathrm{W}_{10} \approx 3$ when $v_{1}^{2}=1124, v_{f}^{2}=0.0015$ and $v=0.6$. With this value of $v$, $\mathrm{W}_{10}=2.11641$ and $\mathrm{w}_{20}=6.3109$.

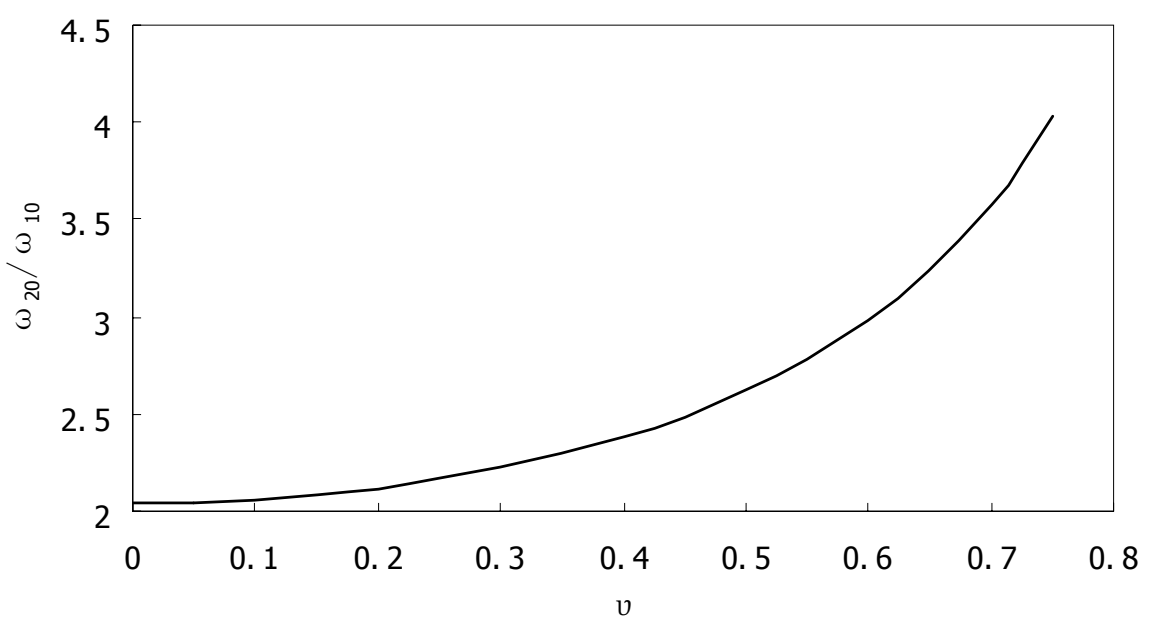

Figure 2. Ratio of transverse natural frequencies versus speed for $v_{1}^{2}=1124$ and $v_{f}^{2}=0.0015$.

\subsection{FUNDAMENTAL RESONANCE AT R NEAR - 10}

In order to obtain the fundamental resonance when the forcing frequency $\mathrm{W}$ is near the first natural frequency $\mathrm{W}_{10}$, one should take $F_{2}=0$ in equation (31). In the following calculation, we take $F_{1}=0.03$ and $n_{\mathrm{c}}=n_{\mathrm{s}}=4$. As equations (30) and (31) do not contain quadratic nonlinear terms, $q_{1}^{w}$ and $q_{2}^{w}$ should not contain even harmonic terms and, thus, can be expressed as

$$
\begin{aligned}
& q_{1}^{w}=A_{11} \cos \left(\mathrm{t}+\mathrm{f}_{11}\right)+A_{13} \cos 3\left(\mathrm{t}+\mathrm{f}_{13}\right)+\cdots, \\
& q_{2}^{w}=A_{21} \cos \left(\mathrm{t}+\mathrm{f}_{21}\right)+A_{23} \cos 3\left(\mathrm{t}+\mathrm{f}_{23}\right)+\cdots
\end{aligned}
$$

where $\mathrm{t}=\mathrm{W} t$ and ${ }^{\mathrm{T} M}$ denote phase difference. The fundamental response is expressed mainly by the $\mathrm{W}-A_{11}, \mathrm{~W}-A_{13}, \mathrm{~W}-A_{21}$ and $\mathrm{W}-A_{23}$ curves which are shown in Figure $3(a),(b),(c)$ and $(d)$, 
respectively. $A_{11}$ and $A_{13}$, defined in equation (33), are respectively the amplitudes of the first and the third harmonic terms in the first variable $q_{1}^{w}$. Meanwhile, $A_{21}$ and $A_{23}$, defined in equation (34), are respectively the amplitudes of the first and the third cosine harmonic terms of the second variable $q_{2}^{w}$. To facilitate the understanding of the relation between forced and free vibration, the free vibration backbone curves for $A_{11}, A_{13}, A_{21}$ and $A_{23}$ are also plotted. It can be seen that $\left|A_{11}\right|$ $>>\left|A_{13}\right|$ and $\left|A_{23}\right|>>\left|A_{21}\right|$. Therefore, the first and third harmonic terms are the major modes of $q_{1}^{w}$ and $q_{2}^{w}$, respectively.

One can note the internal resonance on the amplitudes of the responding modes in Figures $3 \mathrm{a}$ and 3d. Both $A_{11}$ and $A_{23}$ possess three solutions. Their first solutions $A_{11}^{(1)}$ and $A_{23}^{(1)}$ are of different phases, and represent the in-phase and out-of-phase responses, respectively. Starting from $\mathrm{P}_{1} \mathrm{~s}$ in the respective figures, both $\left|A_{11}^{(1)}\right|$ and $\left|A_{23}^{(1)}\right|$ increase with $\mathrm{B}$ to the turning points $\mathrm{P}_{2} \mathrm{~s}$. Before $\mathrm{P}_{2} \mathrm{~s},\left|A_{11}^{(1)}\right|$ $>\left|A_{23}^{(1)}\right|$ and thus the major mode of $q_{1}^{w}$ is the responding mode. At $\mathrm{P}_{2} \mathrm{~S},\left|A_{11}^{(1)}\right|$ jumps down and $\left|A_{23}^{(1)}\right|$ jumps up. Afterwards, $\left|A_{11}^{(1)}\right|$ drops and $\left|A_{23}^{(1)}\right|$ rises slowly. The responding modes switch from the major mode of $q_{1}^{w}$ to that of $q_{2}^{w}$. Though the frequency pertinent to the $A_{23}^{(1)}$ is $3 \mathrm{R} t, A_{23}^{(1)}$ here is not triggered by $F_{2} \cos (3 \mathrm{R} t)$ which acts on $q_{2}^{w}$. Rather, $A_{23}^{(1)}$ is induced by the nonlinear effect intrinsic to the dynamic system. This phenomenon is typically known as internal resonance. Noticeably, the frequencies at $\mathrm{P}_{2} \mathrm{~S}$ are close to the first natural frequency $\mathrm{w}_{10}$ or the exchange of the responding mode occurs at the vicinity of $\mathrm{w}_{10}$.

The second solutions of $A_{11}$ and $A_{23}$ are respectively $A_{11}^{(2)}$ and $A_{23}^{(2)}$ which are of the same phase. $A_{11}^{(2)}$ and $A_{23}^{(2)}$ start from $\mathrm{P}_{4} \mathrm{~S}$ and terminate at $\mathrm{P}_{6} \mathrm{~S}$ via turning points $\mathrm{P}_{5} \mathrm{~S}$. In the course, $\left|A_{11}^{(2)}\right|$ increases gradually but $\left|A_{23}^{(2)}\right|$ decreases gradually. In other words, the responding mode transits gradually from the major mode of $q_{2}^{w}$ back to that of $q_{1}^{w}$. The third solutions of $A_{11}$ and $A_{23}$ are respectively $A_{11}^{(3)}$ and $A_{23}^{(3)}$ which are again of the same phase. $A_{11}^{(3)}$ and $A_{23}^{(3)}$ start from $\mathrm{P}_{7} \mathrm{~s}$ and terminate at $\mathrm{P}_{9} \mathrm{~S}$ via turning points $\mathrm{P}_{8} \mathrm{~s}$. In the course, both $\left|A_{11}^{(3)}\right|$ and $\left|A_{23}^{(3)}\right|$ drop gradually. There is no exchange of responding mode as in the first solutions $\left(A_{11}^{(1)}\right.$ and $\left.A_{23}^{(1)}\right)$ and second solutions $\left(A_{11}^{(2)}\right.$ and $\left.A_{23}^{(2)}\right)$.

The internal resonance pattern for $A_{13}$ and $A_{21}$ revealed in Figures $3 \mathrm{~b}$ and $3 \mathrm{c}$ are similar to that of $A_{11}$ and $A_{23}$. However, $A_{13}$ and $A_{21}$ are only the minor modes of respectively $q_{1}^{w}$ and $q_{2}^{w}$ as $\left|A_{13}\right|$ $\approx\left|A_{21}\right|<<\left|A_{11}\right| \approx\left|A_{23}\right|$. 
To the best knowledge of the authors, the internal resonance phenomenon portrayed in Figures $3 \mathrm{a}$ to $3 \mathrm{~d}$ bodies have not been reported in the literature of axially moving bodies and nonlinear vibration. Nevertheless, the phenomenon is very much similar to the internal resonance previously reported for nonlinear vibration of clamped-hinged beams [28] and thin plates with one edge clamped and the opposite edge hinged [29]. Though they arises from different sets of governing equations and are solved by different methods, they share the common cubic nonlinearity feature and $\mathrm{w}_{20} \approx 3 \mathrm{w}_{10}$.

Figure 4 shows the forced response curves of the Duffing equation as obtained by adding the excitation term $F_{1} \cos \mathrm{W}$ to the right hand side of equation (25). Comparing Figure 4 to Figure 3a, one can discover the important difference between the characteristics of the one and the two degreeof-freedom systems. In the single degree-of-freedom system, $A_{11}^{(1)}$ and $A_{11}^{(2)}$ in Figure 3 a becomes $A_{1}^{(1)}$ in Figure 4. The turning point $\mathrm{P}_{2}$ does not exist and there is no internal resonance for the inphase response curve. On the other hand, the out-of-phase responses $A_{11}^{(3)}$ and $A_{1}^{(3)}$ in the two figures exhibit the same characteristic.

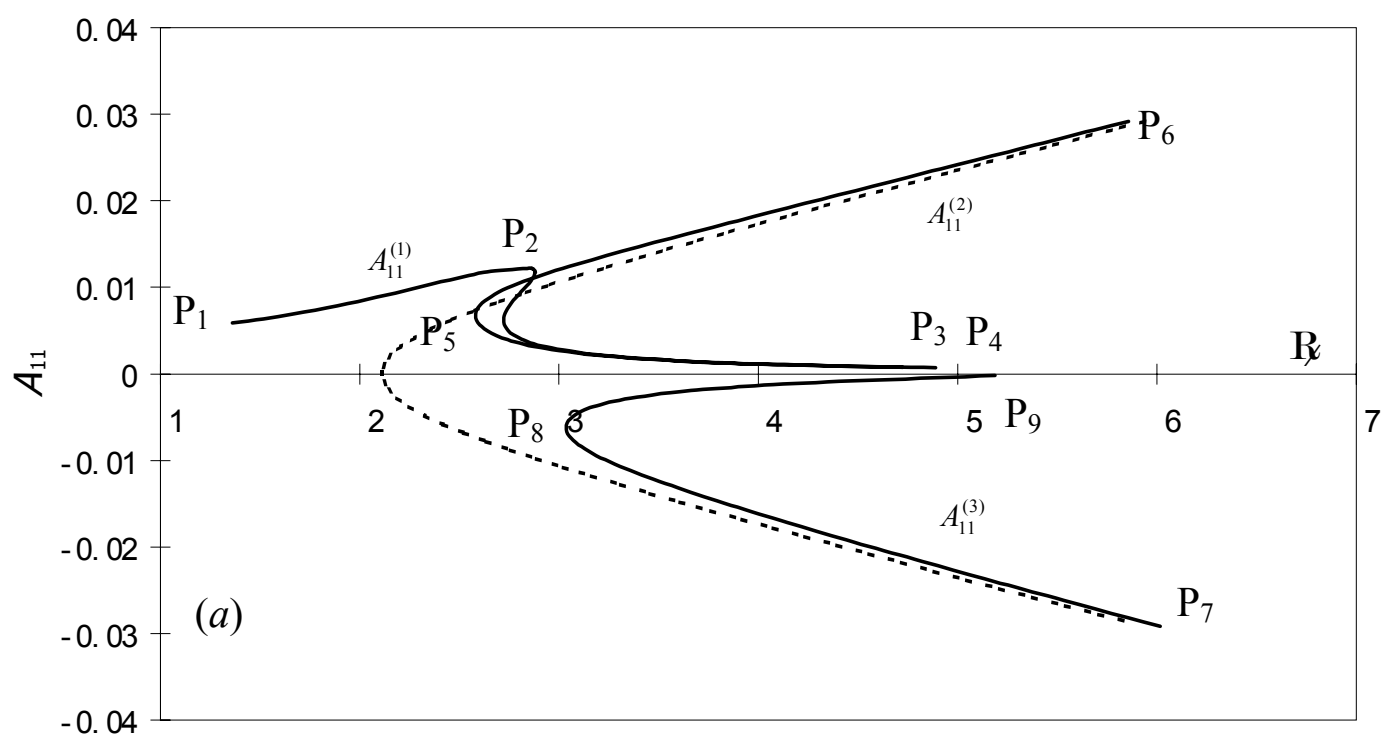

Figure 3a. Fundamental resonance as $\mathrm{W} \approx \mathrm{W}_{10}$, - denotes forced response curve and - - denotes backbone curve. 


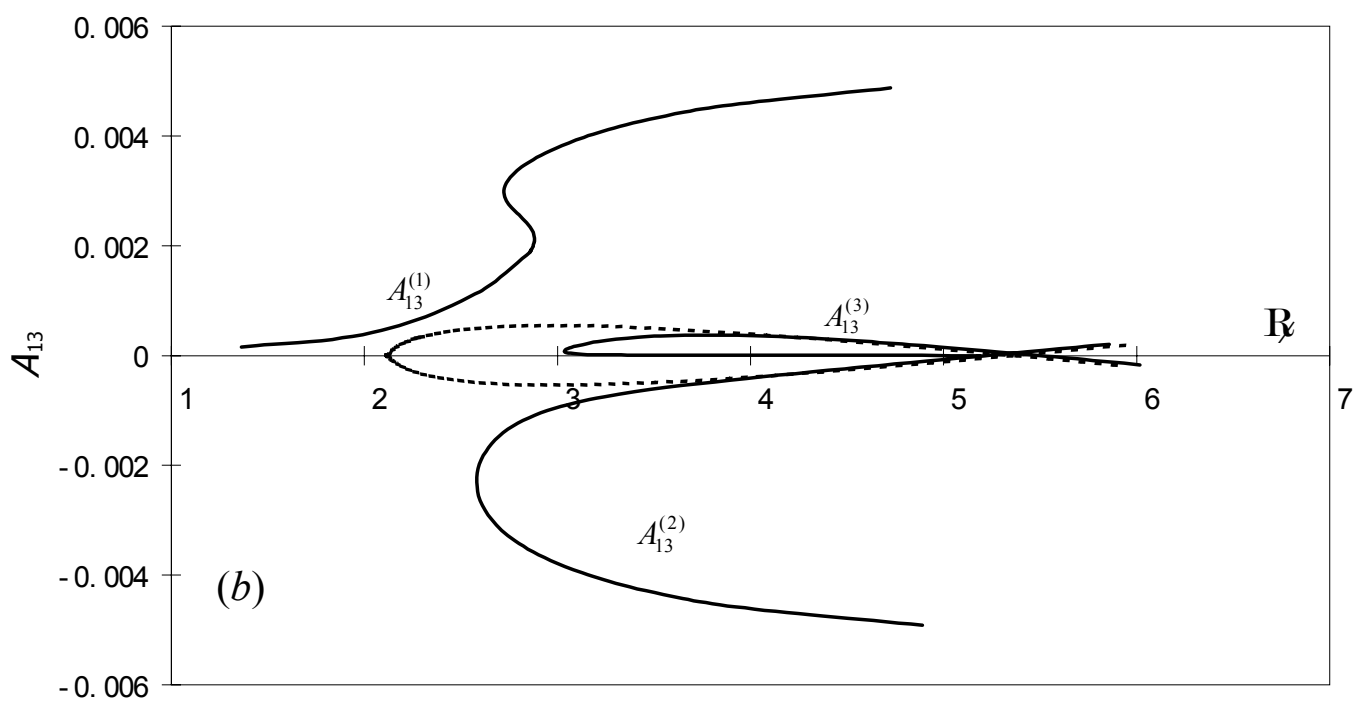

Figure 3 b. Fundamental resonance as $\mathrm{W} \approx \mathrm{W}_{10},-$ denotes forced response curve and - - denotes backbone curve.

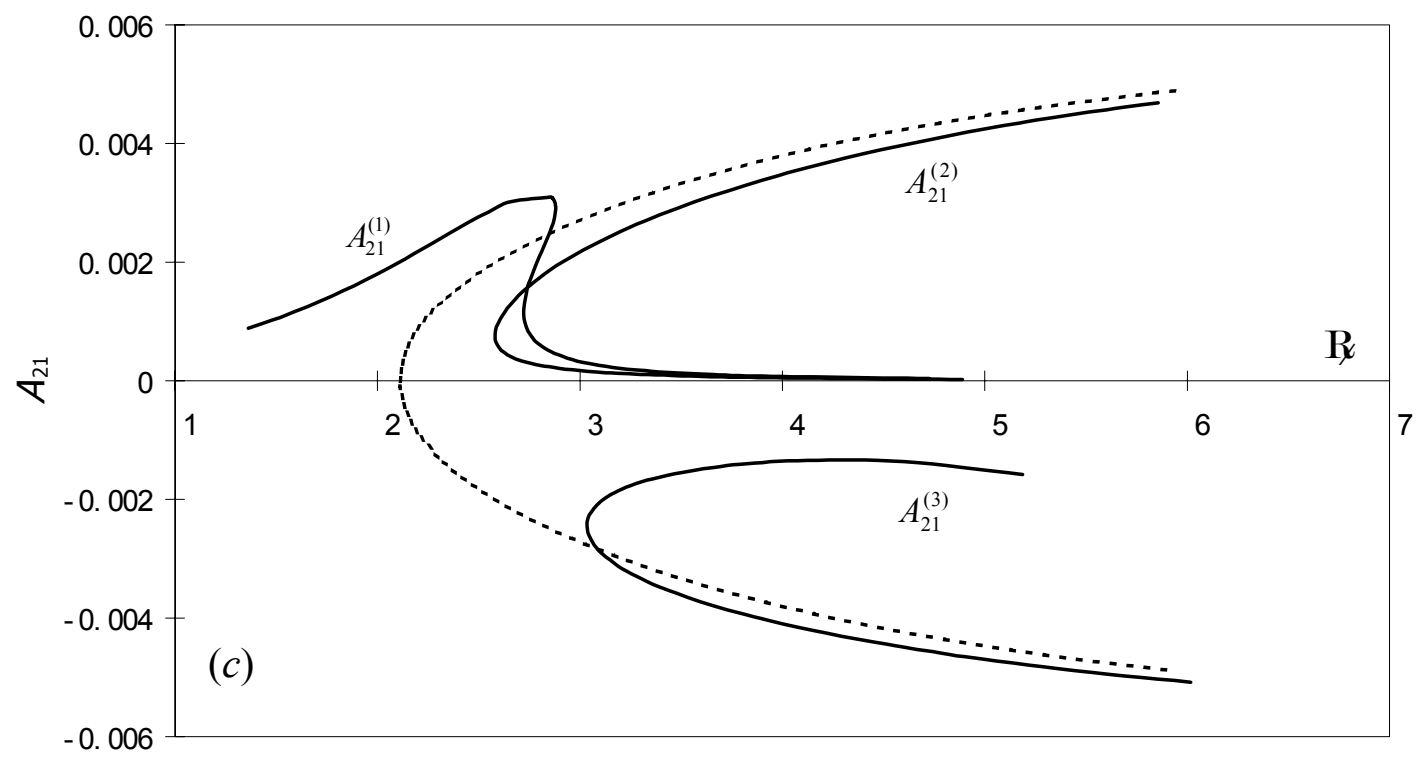

Figure 3c. Fundamental resonance as $\mathrm{W} \approx \mathrm{W}_{10},-$ denotes forced response curve and - - denotes backbone curve. 


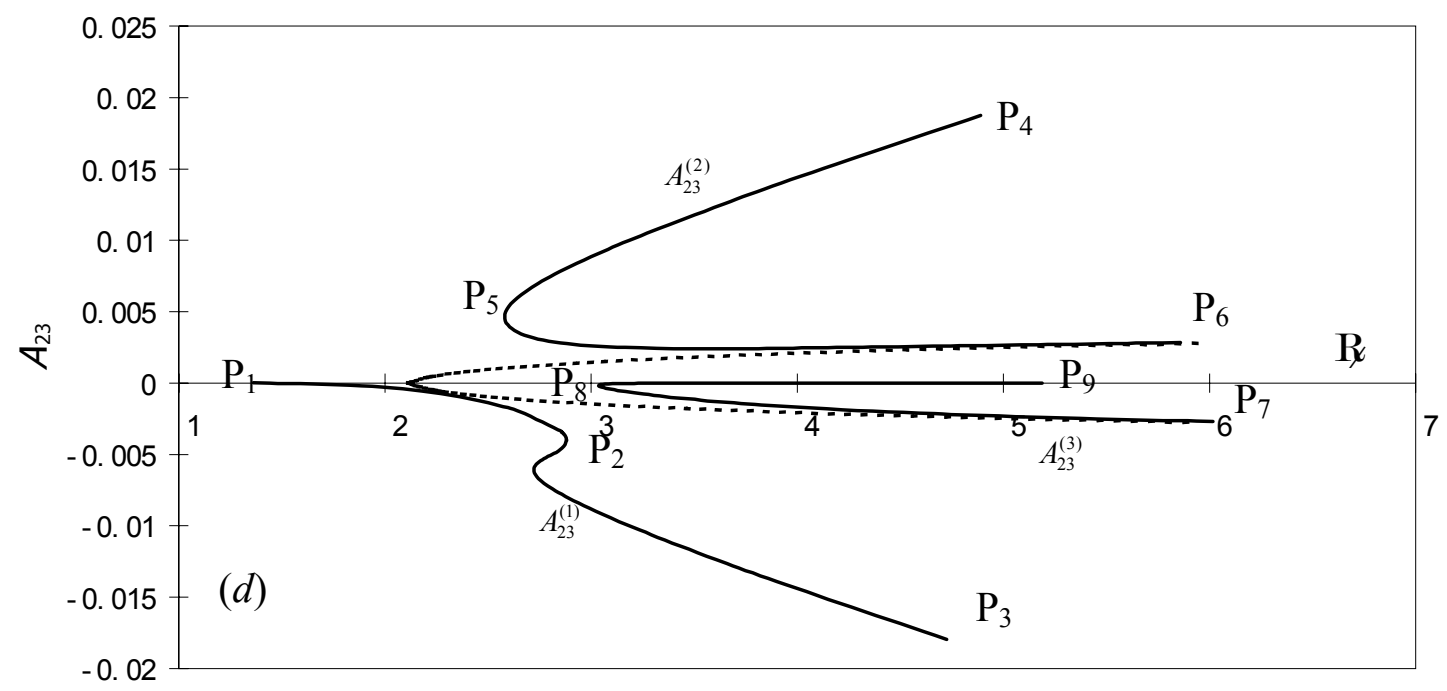

Figure 3d. Fundamental resonance as $\mathrm{W} \approx \mathrm{W}_{10},-$ denotes forced response curve and - - denotes backbone curve.

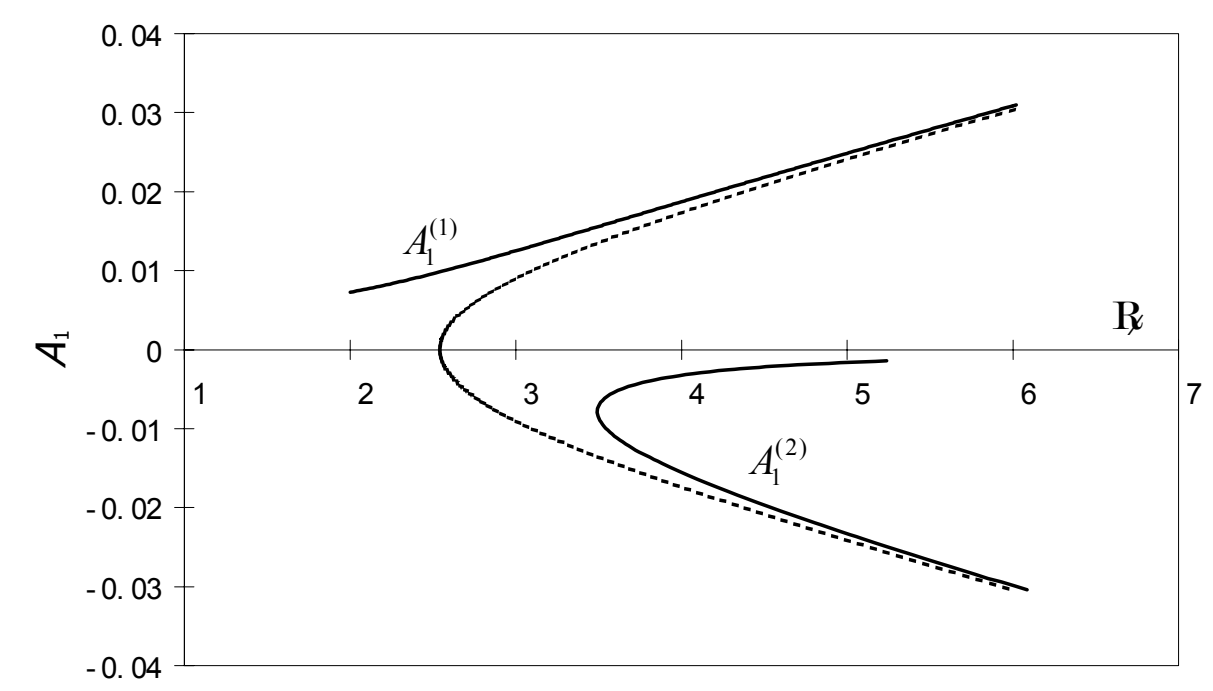

Figure 4. Forced response curve of Duffing equation as $\mathrm{W} \approx \mathrm{W}_{10},-$ denotes forced response curve and - - denotes backbone curve.

\subsection{SUPERHARMONIC RESONANCE AT R NEAR - $10 / 3$}

In order to obtain the superharmonic resonance as $\mathrm{R}$ near $-{ }_{10} / 3$, one should again take a nonzero $F_{1}$ and a zero $F_{2}$ in equations (30) and (31). Furthermore, we set $F_{1}=0.03$ and $n_{\mathrm{c}}=n_{\mathrm{s}}=4$. The solution of $q_{1}^{w}$ and $q_{2}^{w}$ are again taken in the form of equations (33) and (34). Results show $\left|A_{13}\right| \gg>A_{11} \mid$ and $\left|A_{23}\right| \gg>\left|A_{21}\right|$. In other words, the response is dominated by the third harmonic terms whose frequency is three times of the excitation frequency. Consequently, these resonance are called superharmonic resonance which are expressed by the $\mathrm{W}-A_{13}$ and $\mathrm{W}-A_{23}$ curves in 
Figure $5 \mathrm{a}$ and $5 \mathrm{~b}$, respectively. The superharmonic responses of $\mathrm{W}-A_{13}$ and $\mathrm{W}-A_{23}$ show the same characteristics which are similar to that of the fundamental resonance of the single degree-offreedom system in Figure 4. The sharp turns of the frequency-amplitude response curves are responsible for the jump phenomenon.

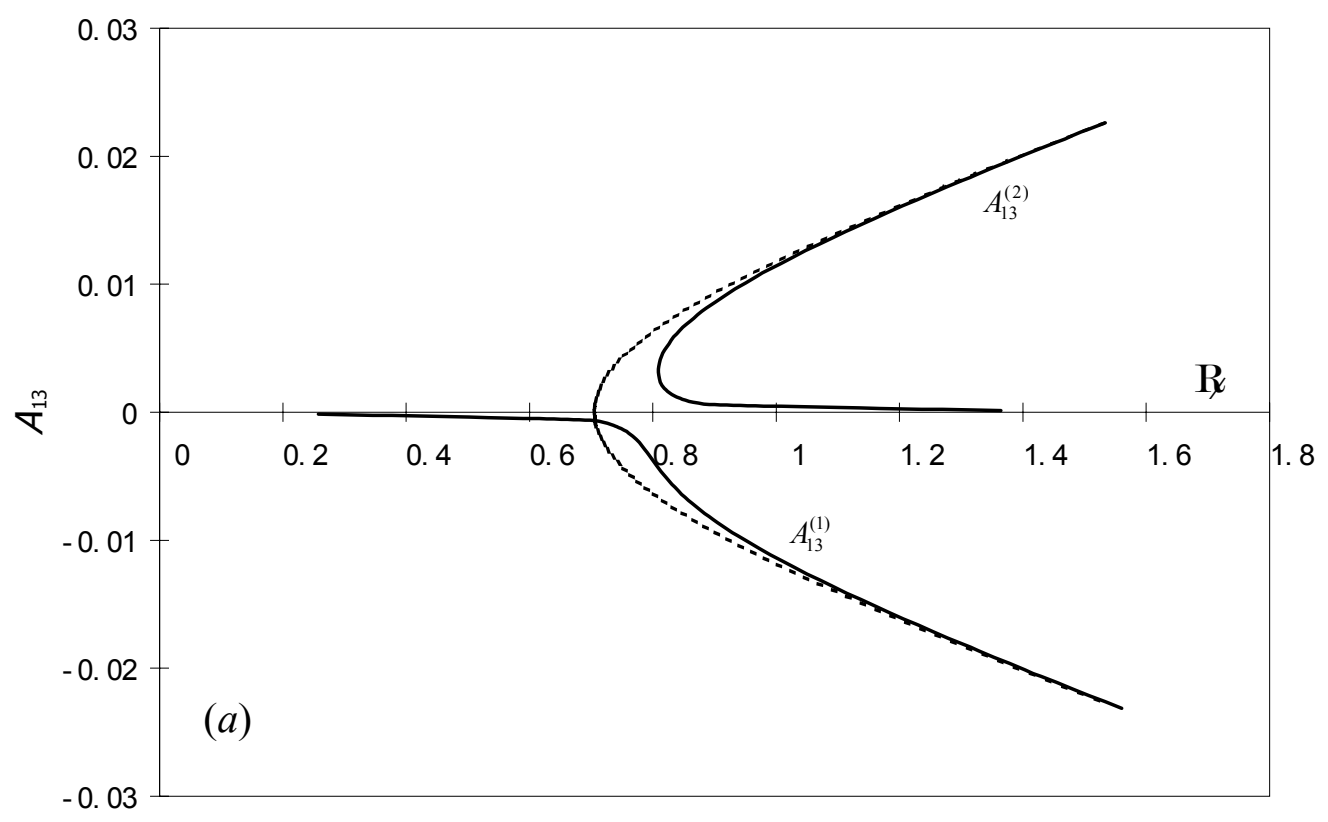

Figure 5a. Superharmonic resonance as $\mathrm{W} \approx \mathrm{W}_{10} / 3,-$ denotes forced response curve and - - denotes backbone curve.

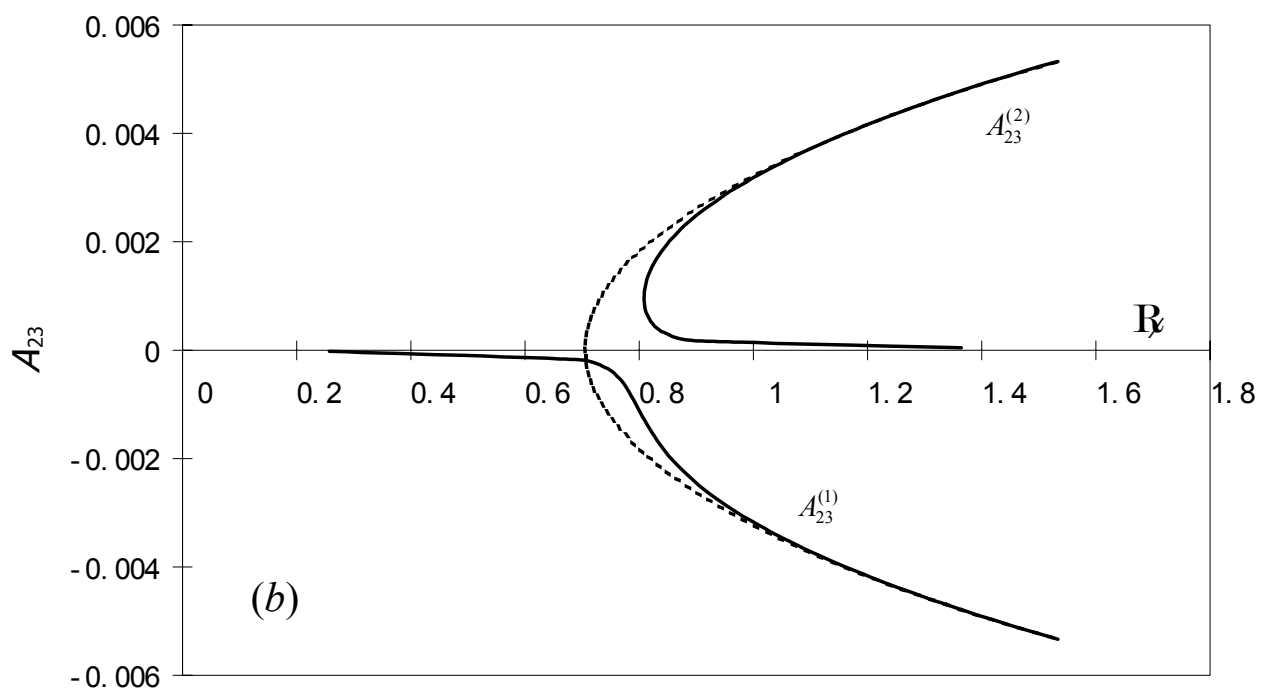

Figure 5 b. Superharmonic resonance as $\mathrm{W} \approx \mathrm{W}_{10} / 3 .-$ denotes forced response curve and - - denotes backbone curve. 


\subsection{FUNDAMENTAL RESONANCE AT R NEAR - 20}

In order to obtain the fundamental resonance as $\mathrm{W}$ nears the second natural frequency $\mathrm{W}_{20}$, one should take a zero $F_{1}$ and a non-zero $F_{2}$ in equations (30) and (31). Furthermore, we take $F_{2}=0.03$ and $n_{\mathrm{c}}=n_{\mathrm{s}}=4$. The solutions of $q_{1}^{w}$ and $q_{2}^{w}$ are also taking the form of equations (33) and (34). Numerical results show that $\left|A_{11}\right|>>\left|A_{13}\right|$ and $\left|A_{21}\right|>>\left|A_{23}\right|$. In other words, the response is dominated by the first harmonic terms whose frequency is the same as the excitation frequency. Therefore, these responses are termed as fundamental resonances. They are expressed by the curves W- $A_{21}$ and W- $A_{11}$ in Figure $6 a$ and $6 b$, respectively. The characteristic of $A_{11}$ and $A_{21}$ is similar to that of the single-degree-of-freedom system shown in Figure 4.

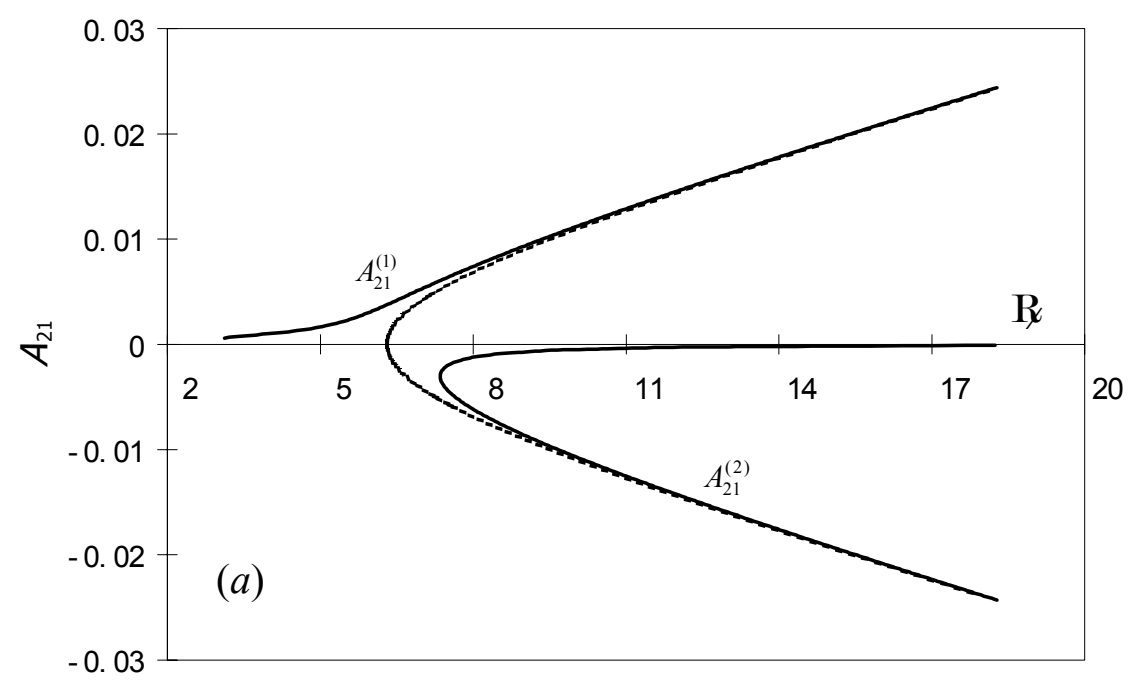

Figure 6a. Fundamental resonance as $\mathrm{W} \approx \mathrm{W}_{20},-$ denotes forced response curve and - - denotes backbone curve.

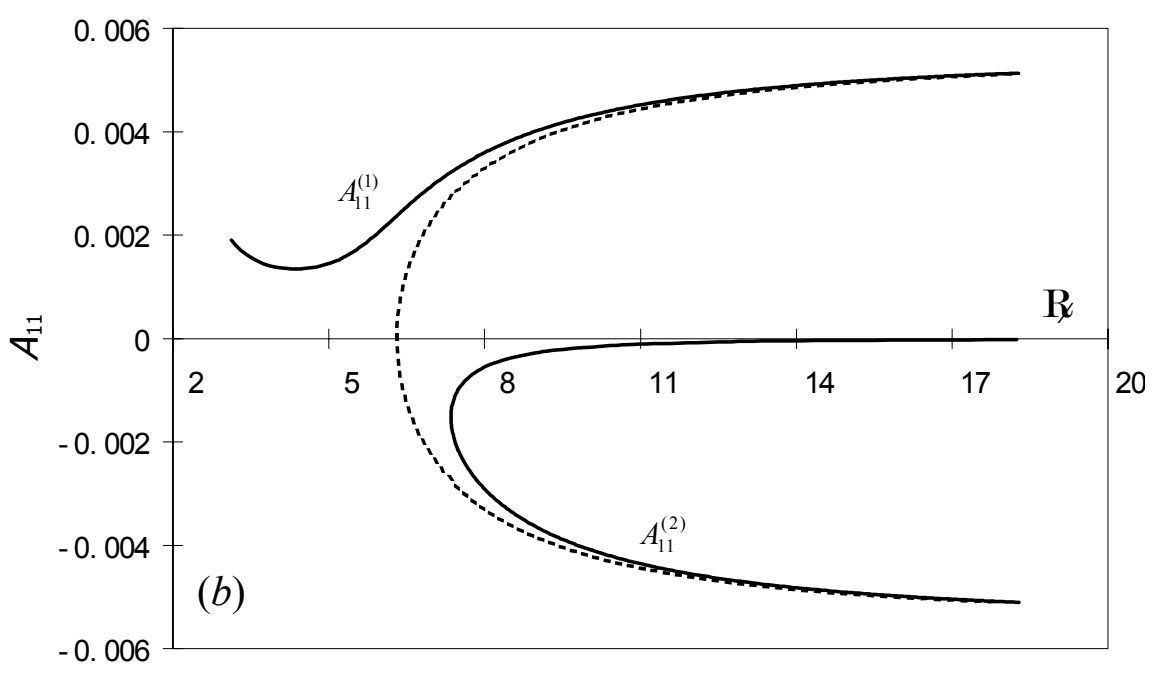

Figure $6 \mathrm{~b}$. Fundamental resonance as $\mathrm{W} \approx \mathrm{W}_{20},-$ denotes forced response curve and - - denotes backbone curve. 


\subsection{SUBHARMONIC RESONANCE AT R NEAR - 20}

Besides the fundamental resonance, there is another kind of resonance named subharmonic resonance that occurs when $\mathrm{W} \approx \mathrm{W}_{20}$. To obtain the subharmonic resonance, one should take a zero $F_{1}$ in equation (30) and a nonzero $F_{2}$ on the third harmonic term in equation (31). Let $t=W t=3 t_{1}$, the solution of $q_{1}^{w}$ and $q_{2}^{w}$ in the present case are taken to be

$$
q_{j}^{w}=\sum_{k=1}^{n_{c}} a_{j k} \cos (2 k-1) \mathrm{t}_{1}+\sum_{k=1}^{n_{s}} b_{j k} \sin (2 k-1) \mathrm{t}_{1} \quad \text { for } j=1,2
$$

Again, we take $f_{2}=0.03$ and $n_{\mathrm{c}}=n_{\mathrm{s}}=4$. Numerical results show that the coefficients $a_{j k}$ and $b_{j k}(\mathrm{j}=1,2, \mathrm{k}=3,4)$ are near to zero. Hence, the assumed solutions are simplified to be

$$
\begin{aligned}
& q_{1}^{w}=A_{11} \cos \frac{1}{3}\left(\mathrm{t}+\mathrm{f}_{11}\right)+A_{13} \cos \left(\mathrm{t}+\mathrm{f}_{13}\right)+\cdots, \\
& q_{2}^{w}=A_{21} \cos \frac{1}{3}\left(\mathrm{t}+\mathrm{f}_{21}\right)+A_{23} \cos \left(\mathrm{t}+\mathrm{f}_{23}\right)+\cdots .
\end{aligned}
$$

Results show that $\left|A_{11}\right|>>\left|A_{13}\right|$ and $\left|A_{21}\right|>>\left|A_{23}\right|$. In other words, the response is dominated by the first harmonic terms whose frequency is one-third of the excitation frequency. Hence, these resonances are called subharmonic resonances which are expressed by the $\mathrm{W}-A_{11}$ and $\mathrm{W}-A_{21}$ curves in Figure 7a and 7b, respectively. In other words, the excitation at a high frequency may produce significant responses in the low frequency modes and, particularly, the fundamental mode. Figures $7 \mathrm{a}$ to $7 \mathrm{~d}$ show how complicated the solution can be when $\mathrm{W} \approx \mathrm{W}_{20}$. Some interesting phenomena are noted below:

(1) There are two solutions in the subharmonic resonance as $\mathrm{W} \approx \mathrm{W}_{20}$.

(2) $\left|A_{11}\right|$ in Figure 7 a can be as large as $6\left|A_{21}\right|$ in Figure $7 \mathrm{~b}, 10\left|A_{23}\right|$ in Figure $7 \mathrm{~d}$ and even $20\left|A_{13}\right|$ in Figure 7c. Clearly, the subharmonic response is dominated by $A_{11}$ or the subharmonic term of the first variable.

(3) Double roots are encountered for $A_{13}$ and $A_{23}$ as shown in Figures 7c and 7d. In other words, $A_{13}^{(1)}=A_{13}^{(2)}, A_{23}^{(1)}=A_{23}^{(2)}$.

(4) There is no jump phenomenon in all subharmonic resonance.

Lastly, it is worth pointing out that one can calculate another set of solutions when $A_{11}=A_{21}=0$. As $\mathrm{W} \approx \mathrm{W}_{20}$, the pertinent response curves $\mathrm{W}-A_{13}$ and $\mathrm{W}-A_{23}$ express the fundamental resonance and are similar to the $\mathrm{W}-A_{11}$ and $\mathrm{W}-A_{21}$ curves in Figures $6 \mathrm{a}$ and $6 \mathrm{~b}$. 


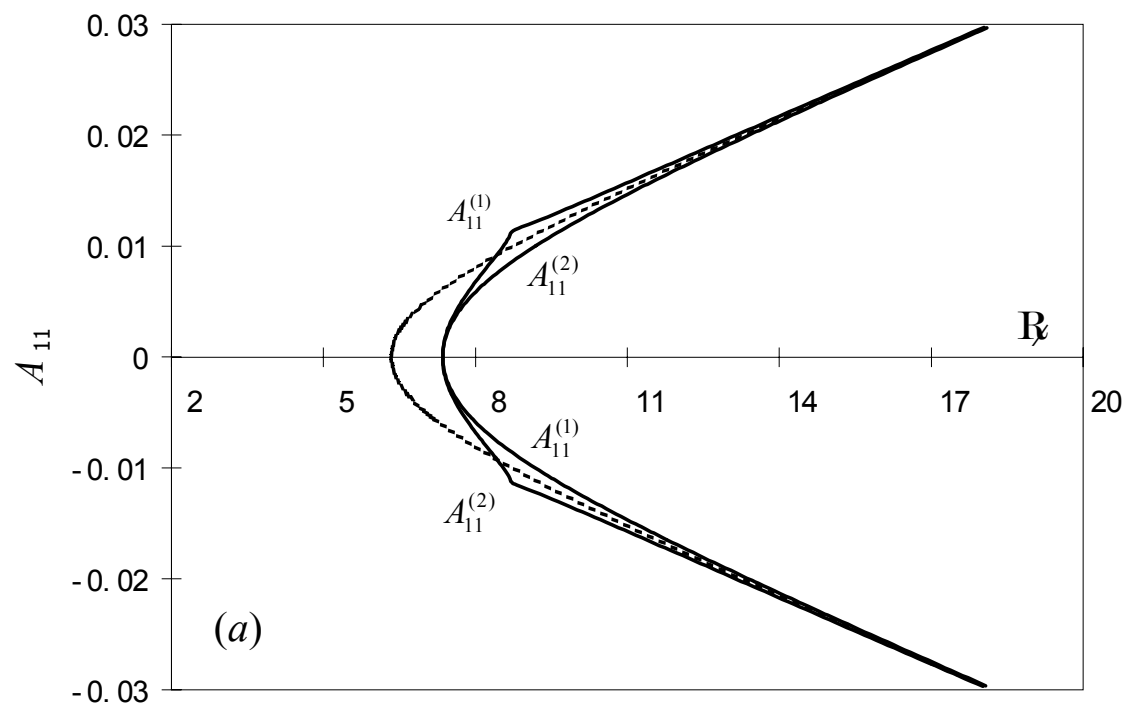

Figure 7a. Subharmonic resonance as $\mathrm{W}_{\mathrm{W}_{20}}$, - denotes forced response curve and - denotes backbone curve.

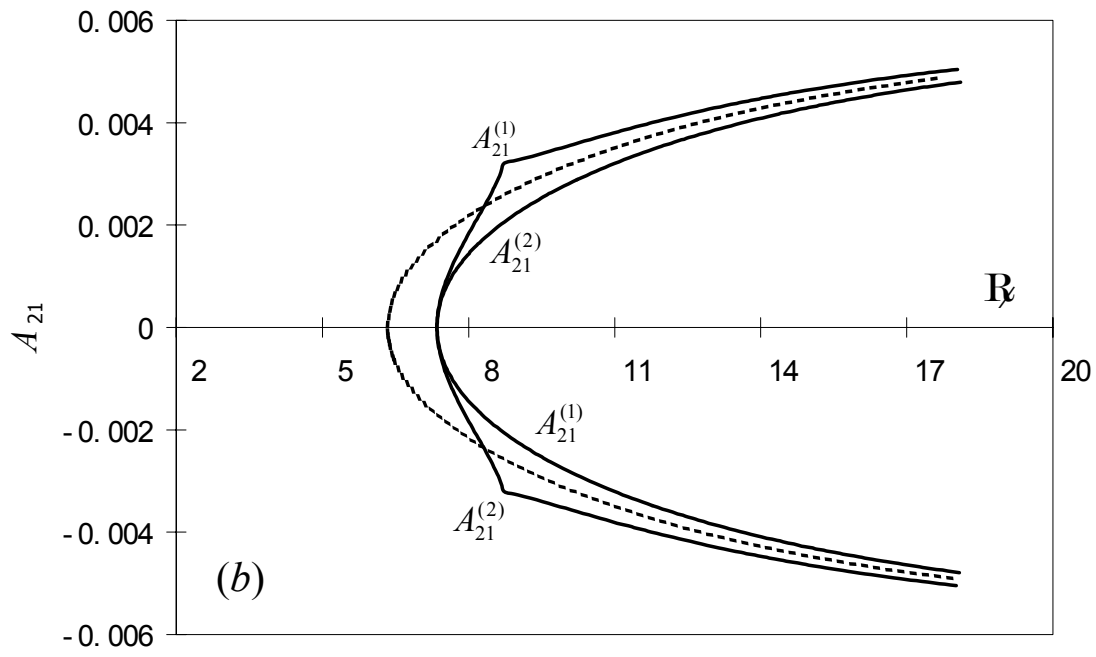

Figure $7 \mathrm{~b}$. Subharmonic resonance as $\mathrm{W} \approx \mathrm{w}_{20},-$ denotes forced response curve and - - denotes backbone curve. 


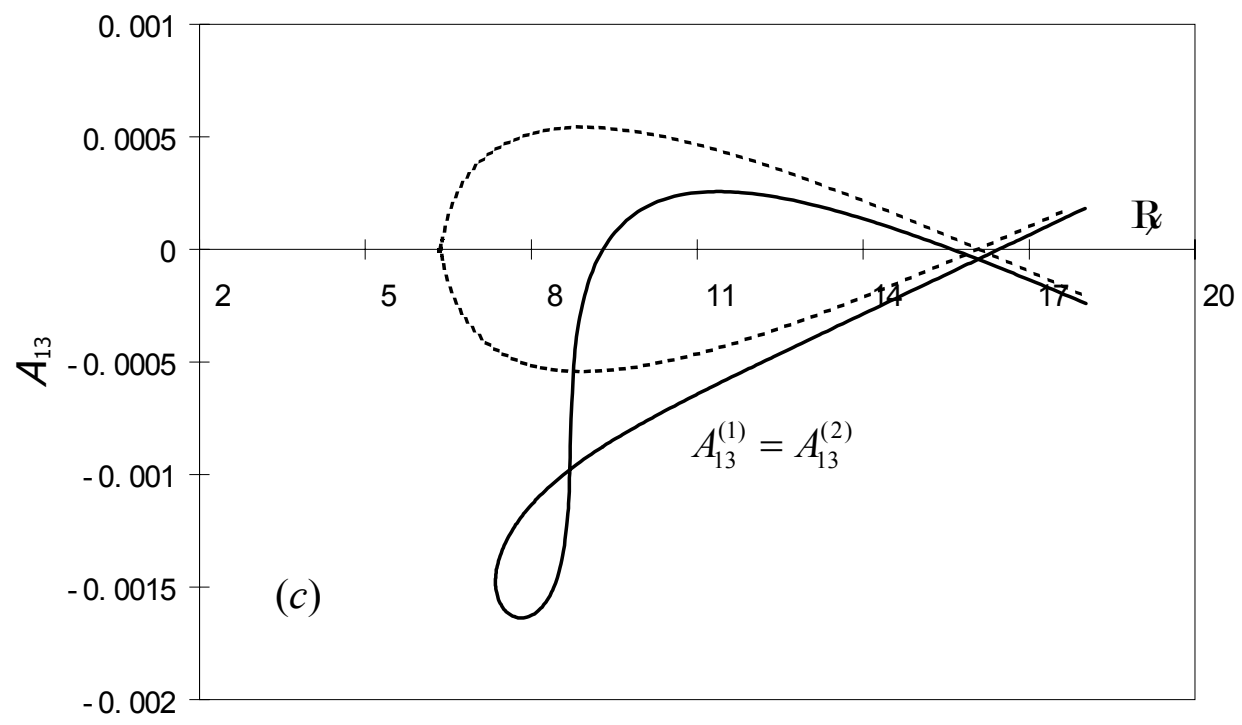

Figure $7 \mathrm{c}$. Subharmonic resonance as $\mathrm{W} \approx \mathrm{w}_{20},-$ denotes forced response curve and - - denotes backbone curve.

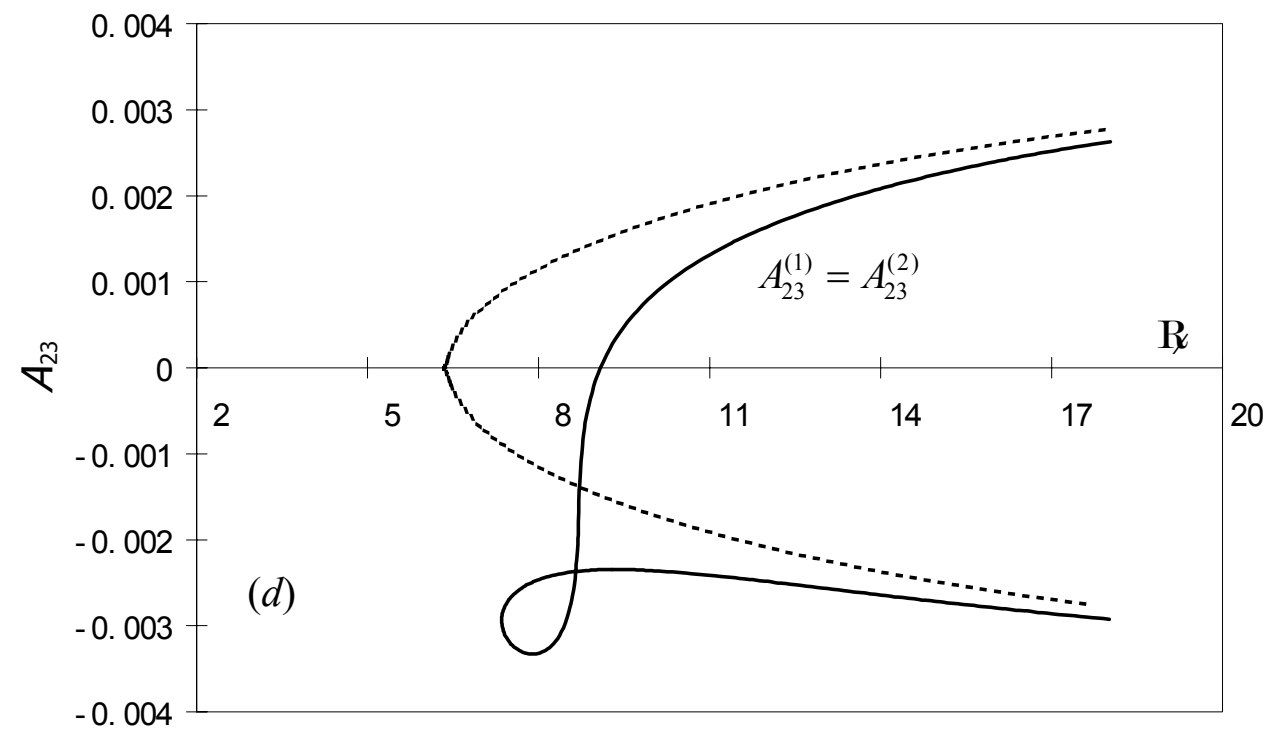

Figure $7 \mathrm{~d}$. Subharmonic resonance as $\mathrm{W} \approx \mathrm{W}_{20},-$ denotes forced response curve and - - denotes backbone curve. 


\section{CONCLUDING REMARKS}

1. The Incremental Harmonic Balance (IHB) method has been shown to be a straightforward, efficient and reliable method for treating the nonlinear vibration of axially moving systems.

2. The forced response of an axially moving beam with internal resonance between the first two transverse modes is studied. In the presence of internal resonance due to the coupling of the two modes, numerical results reveal that excitation at a frequency close to the fundamental frequency can produce a significant response at a higher harmonic frequency. Conversely, excitation at a frequency close to a higher harmonic frequency may produce a significant response at a lower harmonic frequency and, in particular, at the fundamental frequency. The observed internal resonances are rich and complicated. To the best knowledge of the authors, the observations have not been reported in the literature on nonlinear vibration analysis of axially moving media.

3. Stability analysis of the periodic response has not been studied here. It will be discussed in a separate paper.

ACKNOWLEDGMENT - The financial support of the University of Hong Kong in the form of a CRCG Grant is gratefully acknowledged. 


\section{LIST OF REFERENCES}

1. J.A.Wickert and C.D.Mote Jr. 1988 Shock and Vibration Digest 20, 3-13. Current research on the vibration and stability of axially-moving materials.

2. F.Pellicano and F.Vestroni 2000 Journal of Vibration and Acoustics 122, 21-30. Nonlinear dynamics and bifurcations of an axially moving beam.

3. J.A.Wickert and C.D.Mote Jr. 1990 Journal of Applied Mechanics 57, 738-744. Classical vibration analysis of axially moving continua.

4. J.A.Wickert and C.D.Mote Jr. 1991 Journal of Sound and Vibration 149, 267-284. Traveling load response of an axially moving string.

5. J.A Wickert 1992 International Journal of Non-Linear Mechanics 27, 503-517. Non-linear vibration of a traveling tensioned beam.

6. G.Chakraborty, A.K.Mallik and H.Hatal 1999 International Journal of Non-Linear Mechanics 34, 655-670. Non-linear vibration of traveling beam.

7. G.Chakraborty and A.K.Mallik 1999 Nonlinear Dynamics 20, 247-265. Non-linear vibration of a traveling beam having an intermediate guide.

8. A.A.N.Al-Jawi, C.Pierre and A.G.Ulsoy 1995 Journal of Sound and Vibration 179, 243-266. Vibration localization in dual-span, axially moving beams, Part 1: formulation and results.

9. A.A.N.Al-Jawi, C.Pierre and A.G.Ulsoy 1995 Journal of Sound and Vibration 179, 267-287. Vibration localization in dual-span, axially moving beams, Part 2: perturbation analysis.

10. A.A.N.Al-Jawi, C.Pierre and A.G.Ulsoy 1995 Journal of Sound and Vibration 179, 289-312. Vibration localization in band-wheel systems: theory and experiment.

11. J.S.Chen 1997 ASME Journal of Vibration and Acoustics 119, 152-156. Natural frequencies and stability of an axially-traveling string in contract with a stationary load system.

12. C.H.Riedel and C.A.Tan 2002 International Journal of Non-Linear Mechanics 37, 101-116. Coupled, forced response of and axially moving strip with internal resonance.

13. M. Pakdemirli, A.G.Ulsoy and A.Ceranoglu 1994 Journal of Sound and Vibration 169, 179-196. Transverse vibration of an axially accelerating string.

14. M.Pakdemirli and A.G.Ulsoy 1997 Journal of Sound and Vibration 203, 815-832. Stability analysis of an axially accelerating string.

15. E.M.Mockensturm, N.C.Perkins and A.Galip Ulsoy 1996 Journal of Vibration and Acoustics 118, 346-351. Stability and limit cycles of parametrically excited, axially moving strings.

16. L.Zhang, and J.W.Zu 1999 Journal of Applied Mechanics 66, 396-402. Nonlinear Vibration of Parametrically Excited Moving Belts, Part I: Dynamic Response.

17. L.Zhang, and J.W.Zu 1999 Journal of Applied Mechanics 66, 403-409. Nonlinear Vibration of Parametrically Excited Moving Belts, Part II: Stability Analysis.

18. G.Suweken and W.T.Van Horssen 2003 Nonlinear Dynamics 31, 197-223. On the weakly nonlinear, transversal vibrations of a conveyor belt with a low and time-varying velocity.

19. H.R.Öz and M.Pakdemirli 1999 Journal of Sound and Vibration 227, 239-257. Vibration of an axilly moving beam with time dependent velocity.

20. H.R.Öz 2001 Journal of Sound and Vibration 239, 556-564. On the vibration of an axially traveling beam on fixed supports with variable velocity.

21. R.F.Fung and H.C.Chang 2001 Journal of Sound and Vibration 239, 505-514. Dynamic and 
energetic analyses of a string/slider non-linear coupling system by variable grid finite difference.

22. B.Ravindra and W.D.Zhu 1998 Archive of Applied Mechanics 68, 195-205. Low -dimensional chaotic response of axially accelerating continuum in the supercritical regime.

23. J.Moon and J.A.Wickert 1997 Journal of Sound and Vibration 200, 419-431. Nonlinear vibration of power transmission belts.

24. F.Pellicano, A.Fregolent and A.Bertuzzi 2001 Journal of Sound and Vibration 244, 669-684. Primary and parametric non-linear resonance of a power transmission belt: experimental and theoretical analysis.

25. S.L.Lau and Y.K.Cheung 1981 Journal of Applied Mechanics 48, 959-964. Amplitude incremental variational principle for nonlinear structural vibrations.

26. Y.K.Cheung and S.L.Lau 1982 Earthquake Engineering and Structural Dynamics 10, 239-253. Incremental time-space finite strip method for nonlinear structural vibrations.

27. S.L.Lau and Y.K.Cheung and S.Y.Wu 1982 Journal of Applied Mechanics 49, 849-853. A variable parameter incrementation method for dynamic instability of linear and nonlinear elastic systems.

28. S.H.Chen, Y.K.Cheung and S.L.Lau 1989 Journal of Sound and Vibration 128, 13-24. On the internal resonance of multi-degree-of-freedom systems with cubic nonlinearity.

29. S.L.Lau, Y.K.Cheung and S.Y.Wu 1984 Journal of Applied Mechanics 51, 845-851. Nonlinear vibration of thin elastic plates, Part 2: internal resonance by amplitude-incremental finite element. 